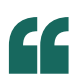

In one

experiment,

we can

produce more new inorganic

materials than

have been

cumulatively

made and

characterized

to date

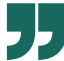

\title{
NANOMATERIALS
}

\section{Printing megalibraries}

Understanding the intimate relation between structure and function drives the discovery and study of new materials. For a long time, such discoveries have been based on a trial-and-error approach in which scientists have studied the effect of incremental changes in a material's structure on its function. Now, writing in Proceedings of the National Academy of Sciences USA, a group led by Chad Mirkin proposes a new approach for the discovery of new nanomaterials in which megalibraries of nanoparticles of different composition and size are simultaneously synthetized and their catalytic activity tested on the fly.

Speeding up the discovery of both new materials and unknown properties of known materials has been one of the major goals in material sciences over the past decade. For example, machine learning and indirect design of materials are two of the computational approaches to pre-screen the immense chemical space with the aim of identifying a handful of possible compounds that can then be synthetized and tested in a lab. Mirkin and co-workers take an alternative approach, scaling down the synthesis and testing so that it is possible to make and test thousands of nanomaterials at once using multiple nanoreactors that occupy just a few square centimetres. Miniaturized multiple-reactors

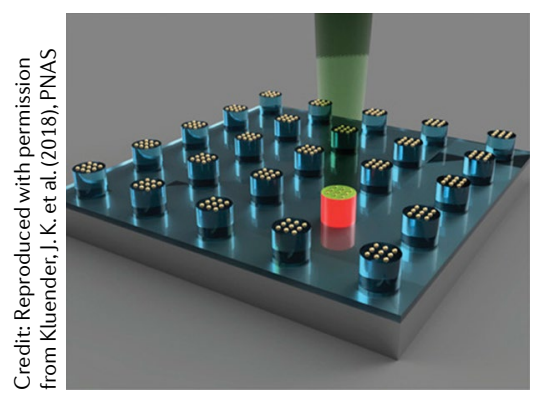

for the synthesis of bulk materials and films have been proposed before; however, things get more complicated at the nanoscale in which, in addition to composition, size affects the final properties of a material.

The group led by Mirkin exploited their scanning probe technique, known as polymer pen lithography (PPL), to create arrays of nanoreactors for the synthesis of nanoparticles of different size and composition. PPL was originally developed to print nanoscale features over square centimetre areas by using arrays of elastomeric pyramidal tips on a flat glass substrate. "We realized that PPL could be turned into a materials discovery tool - in which materials could be explored as a function of both composition and size at unprecedented throughput," explains Mirkin. "For example, commercial PPL tools allow one to print with arrays of as many as 160,000 tips. Each tip can also be used to locally control different chemistries in a tightly confined manner. Because of the small feature sizes, one can pack an enormous number of features into a relatively small area. In one experiment, we can produce more new inorganic materials than have been cumulatively made and characterized to date."

In their current work, Mirkin and co-workers examined the synthesis of $\mathrm{Au}_{x} \mathrm{Cu}_{x-1}$ nanoparticles and their catalytic activity in the synthesis of single walled carbon nanotubes (SWCNTs). Each of the 160,000 tips of a PPL array were spray coated with two metal precursor inks. The radial distribution of the spray coating produces a gradient of each metal, and offsetting the two sprays leads to an array with varying metal compositions. The PPL array was then brought in contact with the substrate for the synthesis of the nanoparticle. Tilting the array can produce a size gradient, so that both composition and size can be varied in the same array. Metal nanoparticles were synthesized by heating the mixture of copolymers to $500^{\circ} \mathrm{C}$.

The catalytic activity of the nanoparticle library was then tested using a high-throughput screening platform - the autonomous research system (ARES) developed by Benji Maruyama and Rahul Rao, co-authors of the paper. In this case, the silicon substrate contains micropillars so that each type of nanoparticle is thermally isolated. In the reaction chamber, the nanoparticle catalysts are exposed to the precursors of SWCNTs (ethene and hydrogen) and then heated by a high energy laser. The same laser was used to collect Raman spectra of the obtained SWCNTs. The study revealed that $\mathrm{Au}_{3} \mathrm{Cu}$, not previously known to catalyze SWCNTs, was the best performing catalyst.

"We used this approach to discover a catalyst for SWCNT synthesis that was not known before, but our study has much broader implications," remarks Mirkin. "We use these methods and the megalibrary approach to both expand and define the materials genome. When one takes into account both composition and size, the parameter space is daunting. We have made particles with as many as eight different elements. Many of these materials have never been contemplated before, let alone in nanoscale form."

Gabriella Graziano

ORIGINAL ARTICLE Kluender, J. K. et al. Catalyst discovery through megalibraries of nanomaterials. Proc. Natl Acad. Sci. USA 116, 40-45 (2018) FURTHER READING Huo, F. et al. Polymer pen lithography. Science 321, 1658-1660 (2008) 